\title{
Intégration d'un processeur cellulaire pour une architecture pyramidale de traitement d'image $\left({ }^{+}\right)$
}

\author{
F. Devos $(*)$, A. Mérigot $\left(^{*}\right)$ et B. Zavidovique (**) \\ (*) IEF, Université Paris Sud, 91405 Orsay, France \\ (**) ADERP-ETCA 1, pl. Frantz Litz, Paris, France
}

(Reçu le 16 mai 1984, accepté le 12 octobre 1984)

\begin{abstract}
Résumé. - Cet article traite de l'intégration d'une architecture pyramidale de multi-processeur cellulaire. Il explique l'intérêt de ce type d'organisation notamment pour le traitement des images, puis présente la manière dont a été implanté un circuit intégré expérimental ainsi que les performances attendues.
\end{abstract}

\begin{abstract}
This paper presents the integration of a cellular multiprocessor pyramidal computer architecture. The interest of such organization, especially for picture processing, is discussed ; then the design of a prototype LSI and its expected performances are detailed.
\end{abstract}

\section{Introduction.}

Le traitement d'images est une branche du traitement de l'information qui présente un intérêt pratique considérable (robotique, météo...), mais aussi de grandes difficultés dans sa mise en œuvre concrète. Celles-ci sont dues principalement à l'énorme quantité d'informations nécessaires au codage numérique d'une image et à la nature répartie, délocalisée de l'information pertinente contenue dans cette image. Notons qu'une image trichrome de $512 \times 512$, codée sur 6 bits pour chaque couleur demande 4,6 Mbits alors que l'information utile pourrait suivant les cas, se réduire de 2 à 3 ordres de grandeur. L'information attachée à un pixel, n'a d'ailleurs que peu d'intérêt en soi, elle devra être analysée, de façon corrélative, pour savoir si ce point appartient à un objet, à un décor ou doit être considéré comme du bruit. C'est un problème classique en traitement de signal, mais le volume des informations à traiter et leur caractère bidimensionnel le rend particulièrement critique [1].

On distingue en général 3 grandes étapes techniques dans le traitement d'image.

- Les transformations d'images (tableaux d'entiers à deux dimensions) en d'autres images (prétraitement, filtrage).

- Les constructions d'autres structures de données,

$\left(^{+}\right)$Communication présentée aux Journées du G.C.I.S., Toulouse les 15 et 16 décembre 1983. d'autant moins volumineuses qu'elles expriment des propriétés plus globales.

- L'analyse de ces propriétés qui conduit à une décision.

Ces traitements mettent en jeu des opérations - ponctuelles (couleur, niveau de gris, seuillage);

- locales (gradient, texture, TCL...).

- globales (moments, projections, transformations diverses, histogrammes...).

\section{Architectures spécifiques.}

Il est évident, qu'en dehors des analyses portant sur les propriétés globales, les machines classiques centralisées, doivent effectuer leurs opérations séquenciellement sur les différents points de l'image et donc effectuer un très grand nombre de fois, le même traitement élémentaire, pour réaliser une étape du traitement global de l'image. C'est évidemment à propos de ce type d'algorithme, naturellement parallélisable, qu'ont été tentées les premières architectures de machines à parallélisme massif et à fonctionnement SIMD [2] : les machines cellulaires du type "Processeurs tableau ". Nous citerons les plus marquantes CLIP 4, DAP, MPP, AAP et renverrons à la liste bibliographique pour des descriptions complètes de ces machines [B-6].

Remarquons cependant que ces machines, de par leur organisation, recopient naturellement la structure de données pour les traitements de bas niveau, et 
qu'une bonne exploitation de cet isomorphisme doit permettre une meilleure organisation du matériel, surtout si les parties opératives des cellules sont organisées avec de faibles largeurs de mots ( 1 ou 2 bits). En effet, dans ce cas les canaux physiques d'entréesortie sont de dimensions réduites et les architectures sont plus simples (intégration plus élevée et fréquence de fonctionnement repoussée). Les calculs arithmétiques de complexité moyenne seront alors organisés en « bit série " et ne pénaliseront pas les performances globales de la machine, s'ils restent réalisables en parallèle sur tout le réseau.

Malheureusement, à mesure que l'on construit des descriptions de l'image, sous forme de propriétés plus globales, leur structure s'apparente de moins en moins à celle d'une pseudo-image, et l'analyse de ces propriétés ne contient plus de possibilités implicites de parallélisation. La machine devient alors relativement inadaptée pour conclure les dernières étapes du traitement. Ce travail est en général rétrocédé à un ordinateur-hôte conventionnel.

Une architecture arborescente pyramidale présente à cet égard, une alternative intéressante à étudier [7]. Cette idée, qui permet de résoudre de manière performante des problèmes de centralisation de données, a déjà été proposée pour certaines applications, notamment les mémoires associatives [8].

Les auteurs des références [9-11] proposent également, pour réaliser des calculateurs d'usage général, une architecture formée par l'interconnexion arboreste de processus où le contrôle est réparti sur tous ces processeurs. Quant au traitement d'image, certains auteurs ont proposé des machines adaptées à un traitement reposant sur une représentation hiérarchisée (multi-résolution) des images [12,13].

Le grand nombre de processeurs qu'implique une approche cellulaire, entraîne des difficultés de réalisation liées au nombre et à la diversité des interconnexions qui existent entre les différentes cellules. C'est pourquoi, nous avons cherché à optimiser le compromis performances/contraintes par les choix architecturaux suivants (Fig. 1).

- A chaque pixel du plan image, est associé un " nanoprocesseur " élémentaire, constitué d'une mémoire locale et d'une unité de traitement (PE). Ces cellules sont organisées en tableau à 2 dimensions, comme CLIP, DAP, MPP, etc... Ce tableau fonctionne en mode SIMD, et possède des communications entre voisins souples et bien adaptées aux problèmes de traitements d'images locaux.

- La centralisation (/diffusion) d'informations, dans les procédures globales sera assurée par un mouvement de " pompe " ascendante (/descendante) dans un arbre binaire de processeurs placés " audessus " du plan image. Tous ces processeurs sont identiques, à ceux du premier plan et peuvent aussi être regroupés en tableaux de processeurs. Chaque tableau fonctionnera alors en mode SIMD et sera géré par un contrôleur unique.

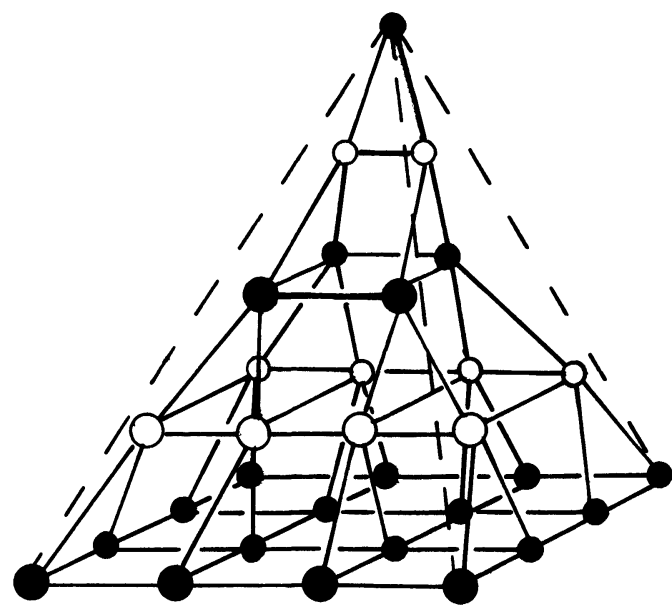

Fig. 1. - Organisation d'une pyramide $4 \times 4$.

[4 $\times 4$ pyramid organization.]

Nous obtenons ainsi une pyramide homogène ayant un fonctionnement global MIMD et deux modes d'interconnexion. Le premier est horizontal, de type "4-connexité ", au sein d'un étage et le second est vertical, de type arbre binaire, entre étages. Remarquons que ce choix de réduction binaire des «PE » à chaque étage, s'il n'est pas minimal en nombre d'étages, compte tenu de la structure plane, simplifie considérablement les interconnexions, et que, malgré tout, une pyramide complète ne demande que le double de «PE " par rapport au processeur tableau de mêmes dimensions.

\section{Architecture et intégration d'un « PE ».}

3.1 Présentation. - La figure 2 résume l'architecture choisie pour le processeur élémentaire. C'est une machine 1 bit qui comprend une unité arithmétique et logique (UAL), une mémoire locale de 64 mots, des registres de travail, des chemins de bus internes et des communications vers un père et quatre voisins. L'architecture est " tourbillonnaire " et tous les mouvements de données passent par l'UAL. Ainsi, quel que soit le mouvement de données, l'organisation temporelle d'une micro-action sera unique et comprendra une "lecture", un "traitement " et une " réécriture " du résultat. Les opérandes peuvent être issus de la mémoire, d'un registre, d'un fils, d'un père ou d'un voisin, sans modification de cycle, de même pour le résultat.

La mémoire, intégrée en local, possède un double accès, totalement indépendant. Elle est adressée, soit directement par le contrôleur, commun à tout un étage, soit par trois pointeurs possédant des primitives d'incrémentation (/décrémentation). Dans un seul cycle, on peut donc disposer simultanément de deux opérandes, les combiner et ranger le résultat. On dispose donc d'une machine à trois adresses effectives. 


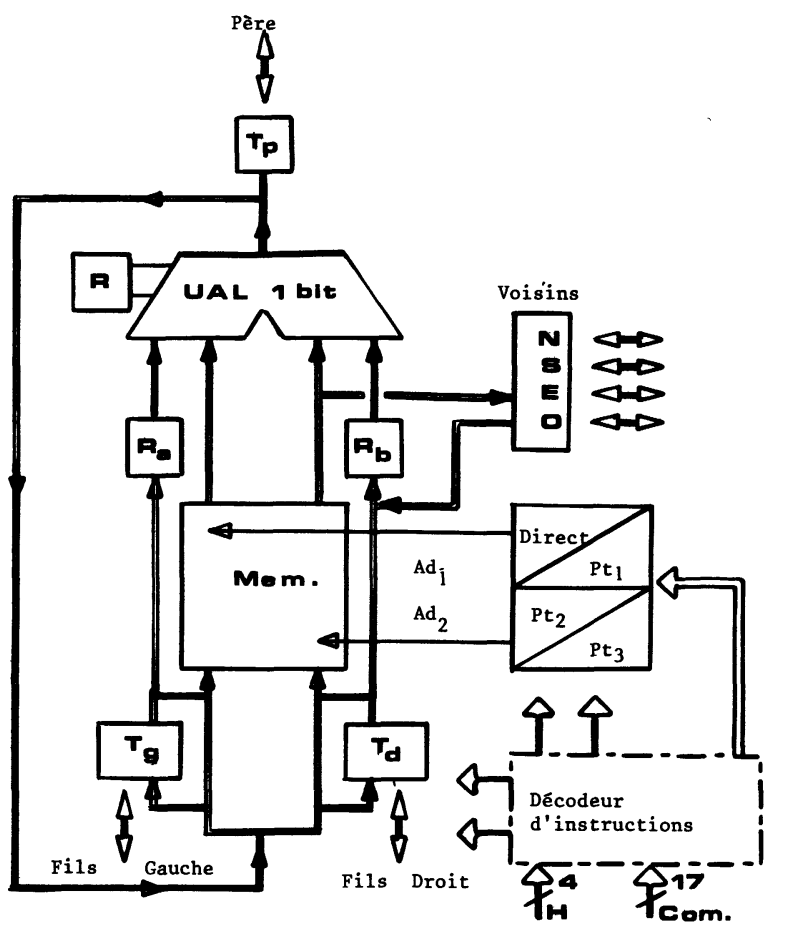

Fig. 2. - Architecture d'un processeur élémentaire. $\left(\mathbf{R}_{\mathrm{a}}\right.$, $R_{b}$ registres $A$ et $\left.B\right)$. $\left(T_{p}, T_{g}, T_{d}\right.$ registres tampon père, fils gauche, fils droit.)

[PE architecture. $R_{a}, R_{b}: A$ and $B$ registers. $T_{p}, T_{g}, T_{d}$ father-latch and son-latchs.]

Remarquons que les connexions disponibles, permettent d'extraire un opérande de la mémoire d'un voisin, de le traiter, puis de le ranger chez un autre voisin, le tout en un seul cycle. Il n'y a bien sûr aucun problème de synchronisme grâce au fonctionnement SIMD de l'étage complet.

Le problème est tout autre pour les communications entre étages car le fonctionnement est dans ce cas MIMD. La difficulté est levée par l'introduction de registres tampons utilisés lors des communications père-fils. La cohérence des données échangées est assurée par un dialogue entre les contrôleurs d'étage.

La machine peut finalement réaliser quatre grandes classes d'instructions :

$$
\begin{aligned}
& \text { - chargements immédiats } \\
& \text { - transferts de données } \\
& \text { - opérations sur ces données } \\
& \text { - instructions sur les pointeurs. }
\end{aligned}
$$

(Les trois premiers types concernent des ressources internes ou externes (voisins), et la dernière agit sur la valeur des pointeurs.)

3.2 InTÉGRATION. - Nous avons voulu, dans une première approche, utiliser le NMOS 5 microns du MPC français, et implanter "au lambda " une maquette de circuit de petite dimension mais permettant d'estimer la faisabilité de l'architecture retenue. Nous avons alors choisi d'intégrer un "PE » et son décodeur. Dans cette étude, le décodeur d'instructions, normalement commun à tout un étage, ne pouvait être " électriquement" dimensionné en vraie grandeur. Nous avons pris le parti de l'implanter de façon très modulaire, semi-automatique. Nous avons enfin préféré les architectures de cellules au fonctionnement le moins critique. Ainsi la mémoire, de petite taille, est statique et d'un schéma maintenant classique (Fig. 3). Les registres sont semi-statiques, et les "latchs", qui ne gardent l'information au plus, qu'un cycle, sont eux, dynamiques. Sans trop rentrer dans les détails de l'implantation, nous dirons que la sélection d'adresses s'obtient à partir de 2 décodeurs de 3 vers 8 et une organisation matricielle $8 \times 8$ de la mémoire. Tous les bus internes sont préchargés et équilibrés, avant lecture, pour éviter les basculements intempestifs de la cellule lue, grâce à un dispositif du type de la figure 4. Les signaux de commande sont amplifiés par des " super-buffers " [14] ou par des « buffers » de type « bootstrap » [15], dans les cas les plus critiques (commande Lec/Ecr ou précharge). Le schéma retenu pour ces derniers est présenté en figure 5.

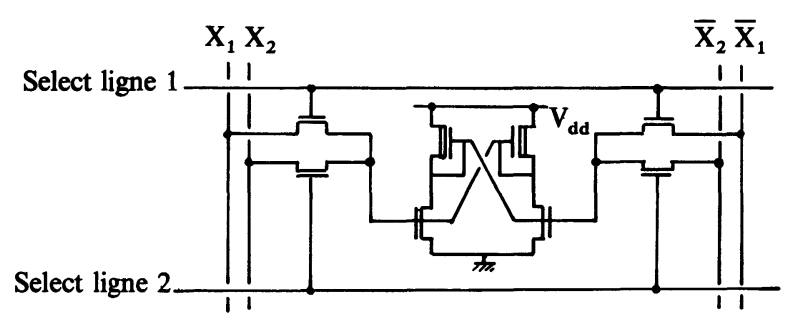

Fig. 3. - Point mémoire double-accès.

[Double-access memory cell]

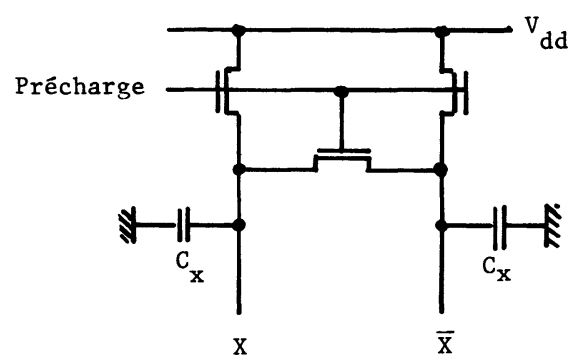

Fig. 4. - Précharge et équilibre des bus.

[Bus precharge and Bus balance.]

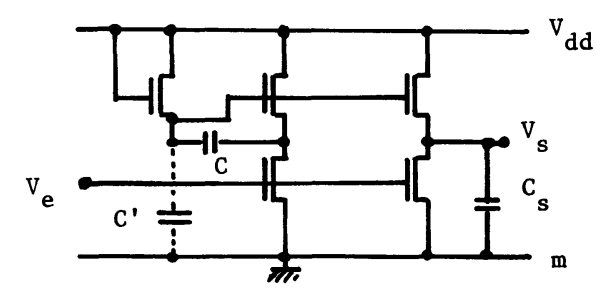

Fig. 5. - Etage « bootstrap ».

[Bootstrap stage.] 
Il demande une consommation importante, mais présente l'avantage d'être de mise au point relativement aisée, même avec une connaissance imprécise des paramètres électriques de la technologie MPC visée.

Le point le plus critique de l'implantation reste la réalisation des amplificateurs de lecture des données. Le schéma de principe est donné en figure 6. C'est un bistable commandé, de fonctionnement très performant mais qui demande des signaux de contrôle très précis, et des charges de bus parfaitement équilibrés [16].

Les pointeurs ont été réalisés en "PLA » montés en compteurs afin de réaliser directement les primitives d'incrémentation et de décrémentation. L'unité arithmétique et le " décodeur d'instructions » ont également été réalisés en «PLA », grâce à GENPLA, un " générateur-simulateur » de PLA [17].

Le placement du circuit a été manuel, en Lucie et l'interconnexion des cellules s'est faite de façon semiautomatique grâce à un logiciel écrit en Lucie-MacroLangage. Les entrées-sorties des données sont réalisées par des plots bidirectionnels 3 états, et les accès aux quatre voisins sont multiplexés. Il y a d'autre part, 14 entrées de commande issues du contrôleur d'étage, et dans cette version, toutes les phases d'horloge sont amenées de l'extérieur pour des raisons évidentes de sûreté de mise en œuvre.

L'ensemble des cellules a été mis au point avec SPICE 2-G4 sur le VAX de l'ENST. Nous avons pu ainsi évaluer les performances temporelles globales escomptées. Celles-ci sont résumées sur le chronogramme de la figure 6. Elles donnent un temps d'accès global à la mémoire de $75 \mathrm{~ns}$ environ (une instruction s'exécutera en $400 \mathrm{~ns}$ ).

Nous avons obtenu pour la surface d'un «PE », $0,8 \times 10 \mathrm{E}+6$ lambda-carré et pour le contrôle d'un étage $1,6 \times 10 \mathrm{E}+6$ lambda-carré. Sur un « $\mathrm{PE}$ », optimisé au micron, on peut espérer réaliser un gain de $30 \%$ en surface, et pour le décodeur, rien qu'en optimisant le PLA, relativement creux, un gain de $50 \%$. On pourrait donc intégrer une pyramide tronquée de 48 «PE» avec ses décodeurs d'instruction, 80 plots bidirectionnels et 40 plots d'entrée (commandes et horloges) sur une seule " puce » ayant une surface d'environ $50 \times 10 \mathrm{E}+6$ lambda-carré. En canaux de 2 microns, cela donne environ un «chip» de $7 \mathrm{~mm} \times 7 \mathrm{~mm}$.

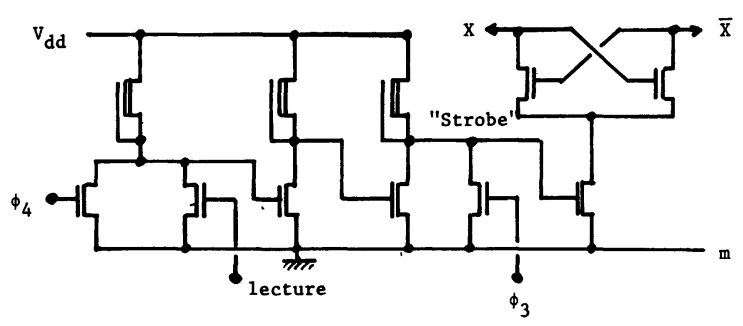

Fig. 6. - Buffer de lecture et génération du strobe.

[Read-buffer and strobe generation.]
Un certain nombre de problèmes risquent néanmoins d'imposer une révision en hausse de ces estimations peut-être optimistes, notamment, celui des communications mais c'est cette raison qui nous a fait envisager l'intégration d'une pyramide tronquée. En privilégiant l'intégration "horizontale" au détriment du vertical, nous n'avons à faire coexister qu'un nombre restreint de niveaux, et les « $\mathrm{PE}$ » se trouvent naturellement au carrefour des axes de communication horizontaux.

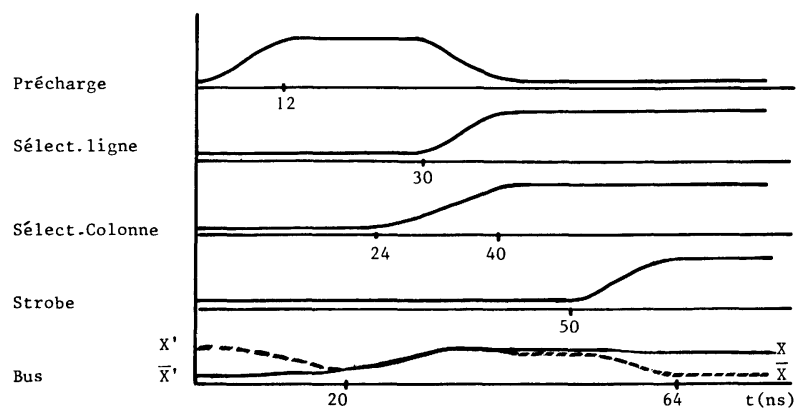

Fig. 7. - Chronogramme d'une lecture dans la mémoire (non compté le temps d'accès au pointeur sélecté).

[Timing of a read memory cycle (without the selected pointer setting time).]
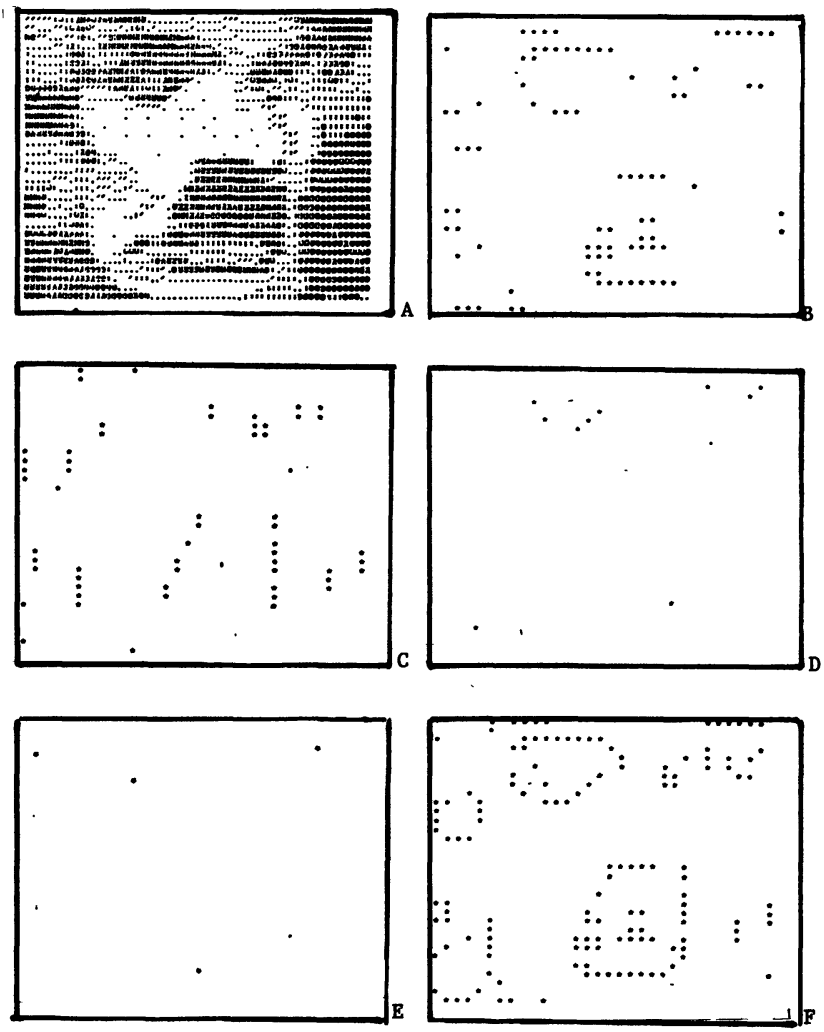

Fig. 8. - Extraction de «contour»: A) image originale (16 niveaux) ; B) C) D) E) « contours » horizontal, vertical, oblique; F) contour final.

[Edge detection : A) Data picture (16 levels); B), C), D), E) horizontal, vertical, diagonal, edges; F) resulting picture.] 
3.3 Evaluation. - Pour évaluer les performances potentielles de la partie opérative d'une machine de traitement d'image basée sur cette architecture, nous avons réalisé une simulation fonctionnelle de la pyramide sur un mini-ordinateur. Un premier exemple d'extraction de contour est décrit en figure 8 a-bc-d-e-f. Il confirme l'efficacité des machines tableaux dans les traitements locaux.

L'algorithme de recherche du pixel de " plus grande valeur " est cependant plus spectaculaire en performance temporelle. Sur une image de 1000 pixels $(32 \times 32)$ à seize niveaux de gris, il ne demande qu'une ascension de la pyramide par bit traité et globalement, il se réalise en 22 cycles grâce à un "pipe-line» des différentes ascensions.

Notons que pour une image de 16000 pixels $(128 \times 128)$, il n'aurait fallu que 4 cycles de plus. D'autres algorithmes ont êté testés [18] et la simulation révèle toujours d'excellentes performances pour les opérations de manipulation d'informations, aussi bien locales, que globales, prouvant ainsi le bienfondé de l'organisation choisie. Pour aboutir à une machine complète, de nombreux problèmes, tant théoriques que pratiques, restent cependant à résoudre. Ceux-ci sont en cours d'exploration, au niveau du contrôleur général de la pyramide, et surtout, au niveau de l'arrangement matériel du plan-mémoire d'alimentation de cette pyramide.

\section{Remerciements.}

Ce travail a été soutenu par le GCIS et l'ETCA/DRET. Nous tenons de plus à remercier M. P. Garda pour ses avis pertinents et MM. Jutand et Dana pour leur aide matérielle informatique.

\section{Bibliographie}

[1] ZAvidoviQue, B., Contribution à la vision des robots, Thèse d'Etat, U.T. Compiègne, Juin 81.

[2] FlynN, M. J., Some Computer Organisation and their Effectiveners, IEEE Trans. Comp. 21-9 (1972) 948-960.

[3] DuFf, M. L. B., CLIP4, A Large Scale Integrated Circuit Array Parallel Processor, Proc. 3rd ICPR 1976, p. 728-733.

[4] Marks, P., Low Level Vision using an Array Processor, CGIP 14 (1980) 281-292.

[5] Batcher, K. E., Design of a Massively Parallel Processor, IEEE Trans. Comp. C $29-9$ (1980) 836-840.

[6] Kondo, et al., An LSI Adaptative Array Processor, IEEE J. Solid state Circuit, Vol. SC 18-2 (1983) 147-156.

[7] MERIGOT, A., Une architecture pyramidale d'un multiprocesseur cellulaire pour le traitement d'images, Thèse $3 \mathrm{e}$ cycle, Orsay, Nov. 1983.

[8] Forster, C. C., Content Adressable Parallel Processor, (Van Nostrand Reinold) 1976.

[9] Mago, G. A., A Cellular Language-directed Computer Architecture, Proc. COMPCON. 1980, p. 179-187.
[10] Browning, S. A., A Tree Machine, Lambda 2nd Quart. 1980, p. 31-36.

[11] SequIN, C. H., Single Chip Computers, the New VLSI Building Blocks, Proc. Caltech Conf. on VLSI 79, 435-446.

[12] Uhr, L., Converging Pyramids of Arrays, Proc. CAPAIDM 81, p. 31-34.

[13] Dyer, C. R., A VLSI Pyramid Machine for Hierarchical Image Processing, Proc. PRIP 1981, p. 381386.

[14] Mead Conway, Introduction to VLSI Design (AddisonWesley) 1980.

[15] ElmaSRY, R., Digital Integrated Circuits, (IEEE Press) 1981.

[16] Howes Morgan, Large Scale Integration, (J. Wiley) 1981.

[17] Garda, P., SAVAry, D., GENPLA, manuel d'utilisation, Rapport Interne IEF, Univ. Paris-Sud.

[18] Merigot, et al., Pyramidal Algorithms for Image Processing, Proc. 7 ICPR 84, p. 828-831. 